Scientific Journal of Hamadan Nursing \& Midwifery Faculty - ISSN 2008-2819

\title{
Effectiveness of Group Therapy Based on Acceptance and Commitment on Social and Health Adjustment of Nursing Students
}

\author{
Leyla Noori ${ }^{1}$, Amene Moradi Shakib ${ }^{2}$, Elmira Ezazi Bojnourdi ${ }^{3}$, Fakhteh Adib $^{4 *}$, Jamal Ashoori ${ }^{5}$ \\ 1. MA of General Psychology, Department of Psychology, Garmsar Branch, Islamic Azad University, Garmsar, \\ Iran \\ 2. MA of Clinical Psychology, Department of Psychology, University of Kharazmi, Tehran, Iran \\ 3. MA of Family Counseling, Department of Counseling, University of Kharazmi, Tehran, Iran \\ 4. Department of Nursing, College of Nursing and Midwifery, Islamic Azad University, Kazeroun Branch, \\ Kazeroun, Iran \\ 5. PhD Student of Psychology, Department of Psychology, Isfahan (Khorasgan) Branch, Islamic Azad University, \\ Isfahan, Iran
}

\begin{tabular}{ll}
\hline Article Info & Abstract \\
\hline
\end{tabular}

Received: $\quad$ 2017/03/03

Accepted: $\quad$ 2017/06/24

Published Online 2017/07/17

DOI: $10.30699 /$ sjhnmf.26.5.3

Original Article

Use your device to scan and read the article online

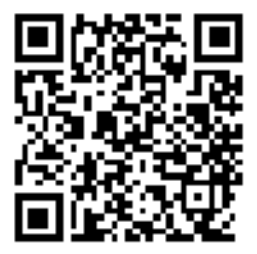

Introduction: University students have a lot of problems in the field of adjustment especially social and health adjustment. Therefore, present research aimed to investigate the effectiveness of group therapy based on acceptance and commitment on social and emotional adjustment of nursing students.

Methods: This research was carried out as a semi-experimental, with a pre-test and post-test design. The statistical population included all nursing students of Islamic Azad University of Pishva branch in 2015-16 academic year. 40 nurses were selected by voluntary sampling method and randomly assigned to two equal groups. The experimental group experienced 8 sessions of 90 minutes of education by group therapy based on acceptance and commitment. Groups completed the dimensions of social and health adjustment of Bell's adjustment questionnaire. Data were analyzed using SPSS19 and by multivariate analysis of covariance method.

Results: The findings showed there was a significant difference between experimental and control groups in the social and health adjustment. In the other words, group therapy based on acceptance and commitment significantly led to improve the social and health adjustment of nursing students $(P \leq 0 / 05)$.

Conclusion: The results are due to the importance of group therapy based on acceptance and commitment on increasing social and health adjustment of nursing students. Therefore, it is suggested that counselors and therapists improve the dimension of adjustment especially social and health adjustment using group therapies based on acceptance and commitment.

Keywords: Group Therapy, Acceptance and Commitment, Social Adjustment, Health Adjustment, Nursing Students

Copyright $($ C 2018, Sci J Hamadan Nurs Midwifery Fac. This is an open-access article distributed under the terms of the Creative Commons Attribution-noncommercial 4.0 International License which permits copy and redistribute the material just in noncommercial usages, provided the original work is properly cited.

How to Cite This Article:

Noori L, Moradi Shakib A, Ezazi Bojnourdi E, Adib F, Ashoori J. Effectiveness of Group Therapy based on Acceptance and Commitment on Social and Health Adjustment of Nursing Students. Sci J Hamadan Nurs Midwifery Fac. 2018; 25 (5): 172-179 


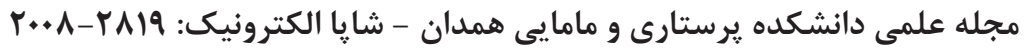

مقاله يثوهشى

\section{اثربخشى گروهدرمانى مبتنى بر يذيرش و تعهد بر سازگًارى اجتماعى و بهداشتى

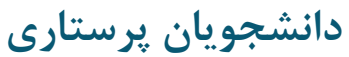

\section{ليلا نورى'، آمنه مرادى شكيب'، الميرا اعزازى بجنوردى"، فاخته اديبَ"، جمال عاشورىه}

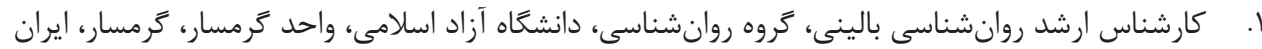

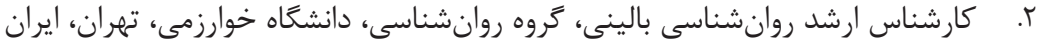

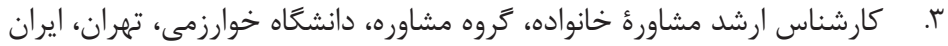

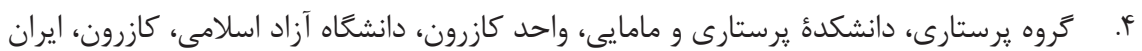

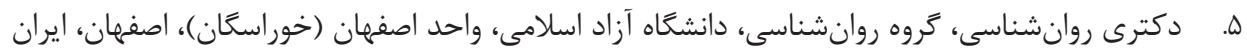

\begin{tabular}{|c|c|c|}
\hline & & \\
\hline \multicolumn{2}{|c|}{ 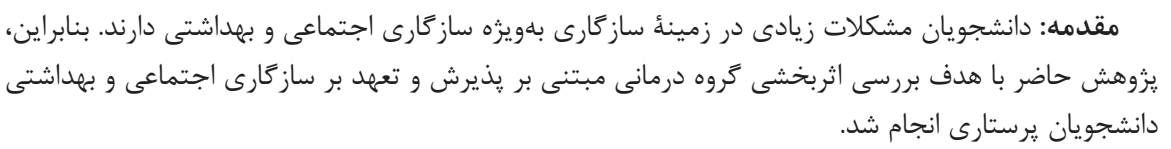 } & تاريخ يذ وصول: \\
\hline \multicolumn{2}{|c|}{ 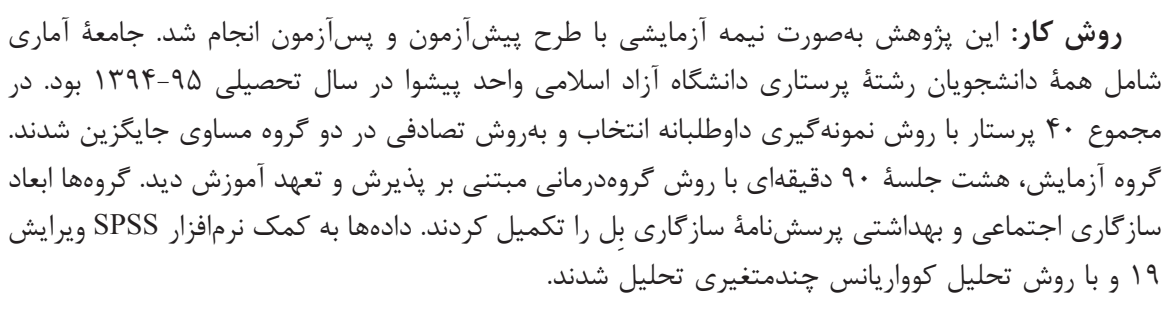 } & 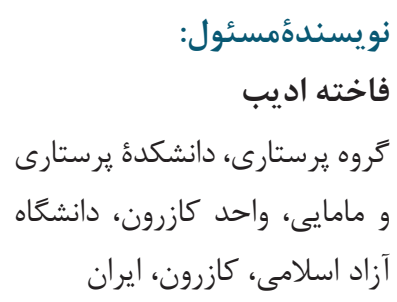 \\
\hline \multicolumn{3}{|c|}{ 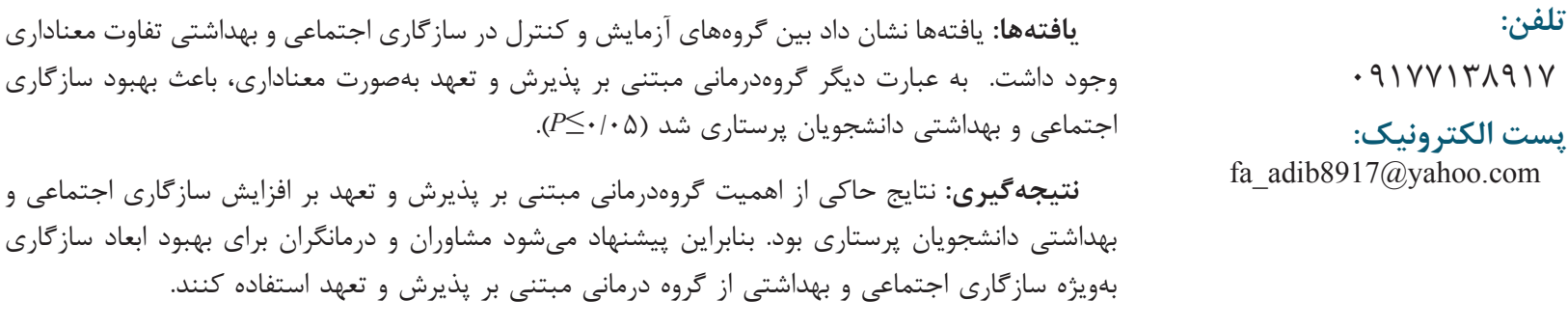 } \\
\hline \multicolumn{3}{|c|}{ وازههاى كليدى: كروه درمانى، يذيرش و تعهد، ساز كارى اجتماعى، ساز كارى بهداشتى، دانشجويان برستارى } \\
\hline 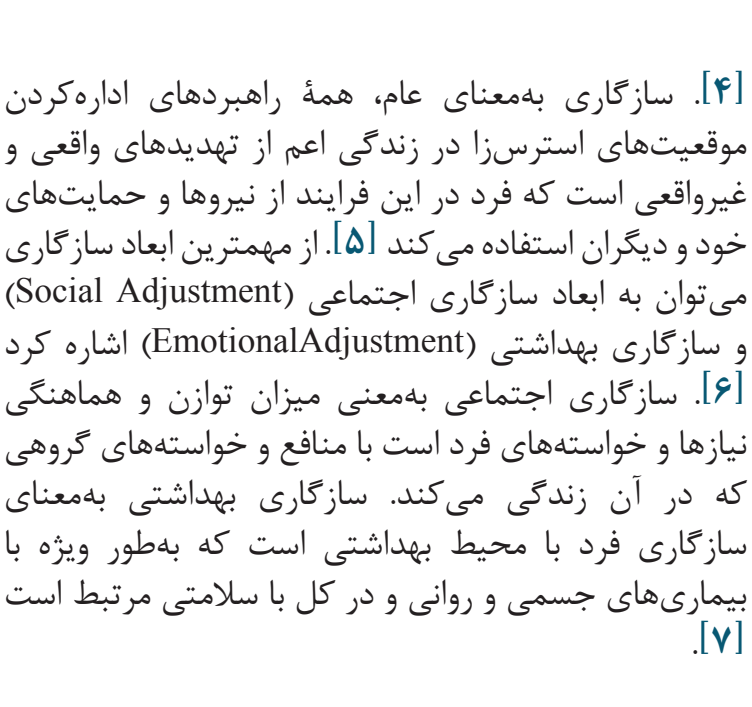 & 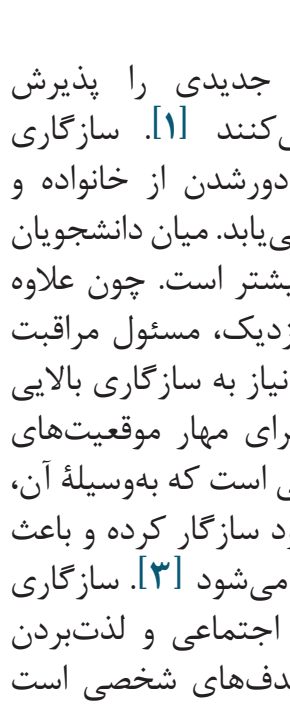 & 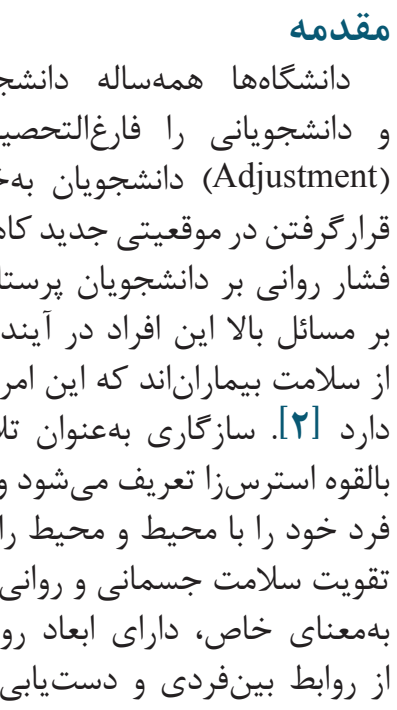 \\
\hline
\end{tabular}


عنوان اثربخشى درمان مبتنى بر يذيرش و تعهد بر سازكارى

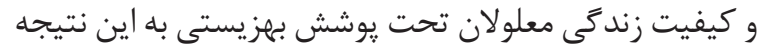

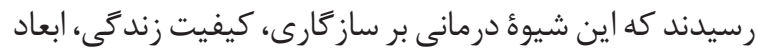

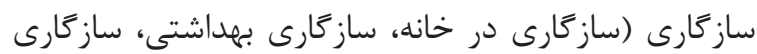

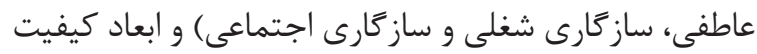

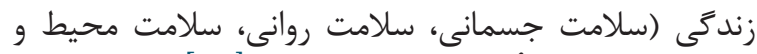

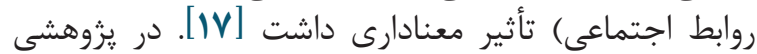

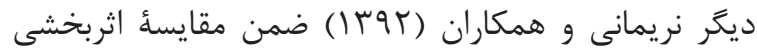

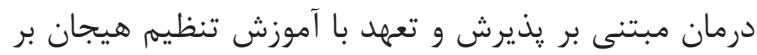

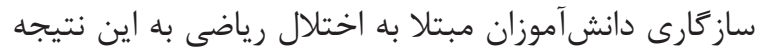

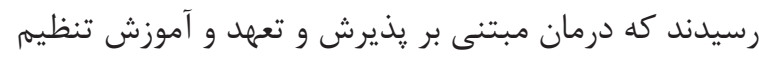

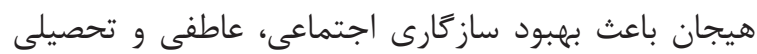

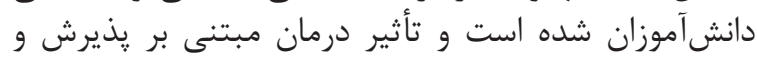
تعهد بيشتر از آموزش تنظيم هيجان بود [11]

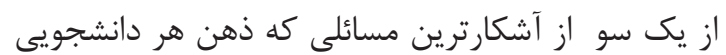

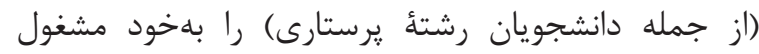

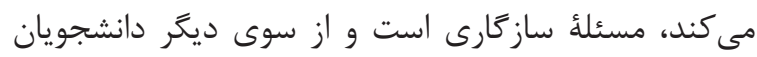

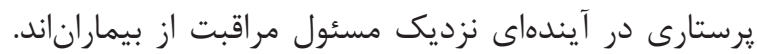

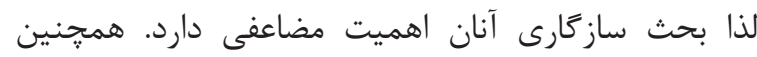

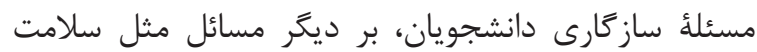

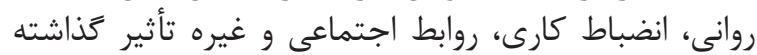

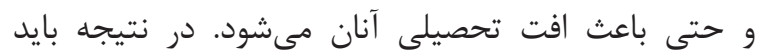

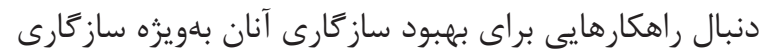

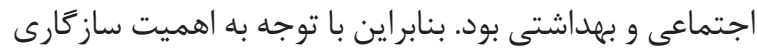

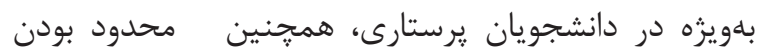

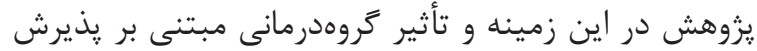

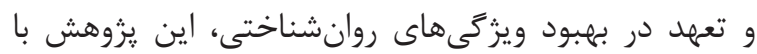

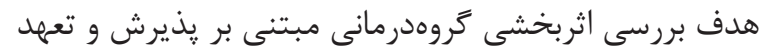

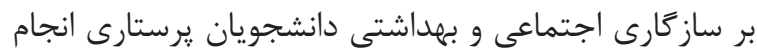

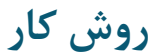

يزوهش حاضر بهصورت نيمه آزمايشى با طرح بيش آزمون

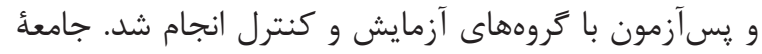

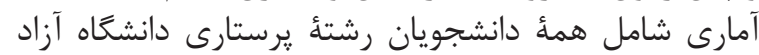

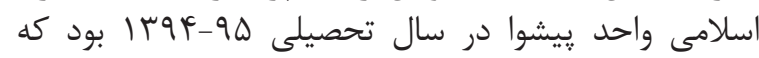

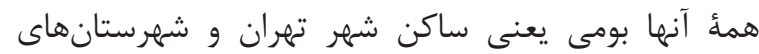

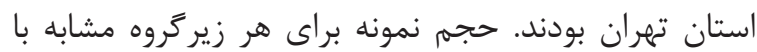

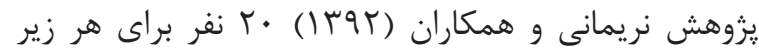

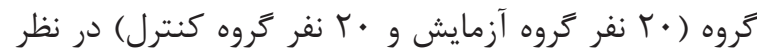

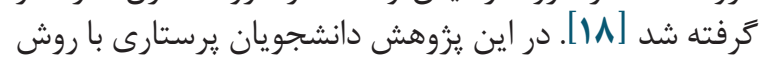

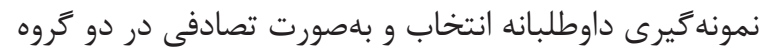

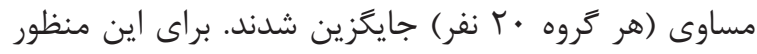

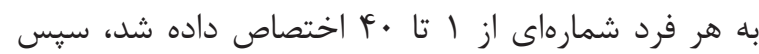

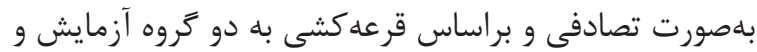

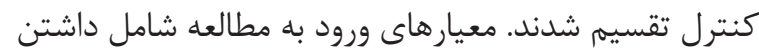

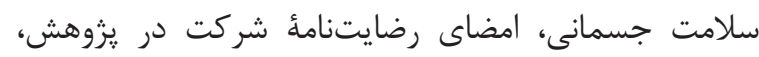

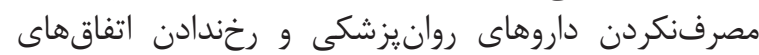
تنشزا مثل طلاق، مركى عزيزان و غيره در شش مان ماه كذشته

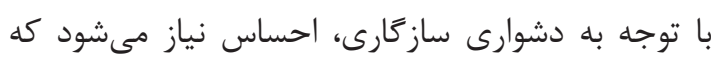

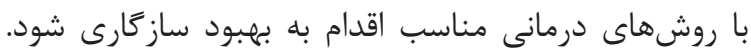

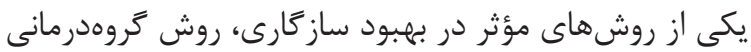
مبتنى بر يذيرش و تعهد (1) Acceptance and Commitment)

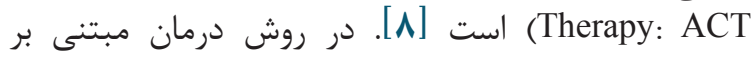

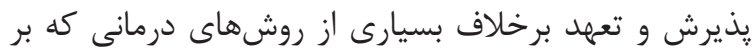

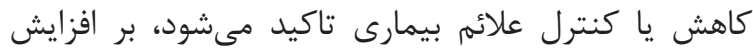

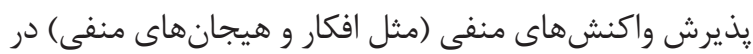

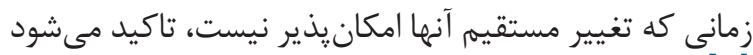

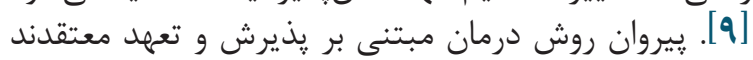

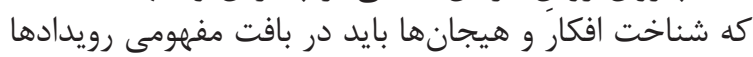

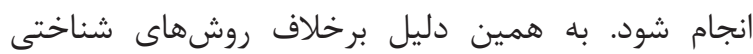

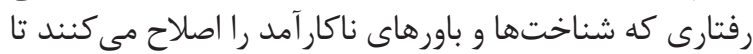

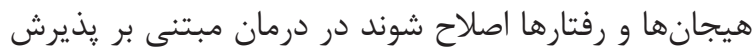

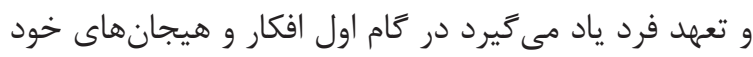

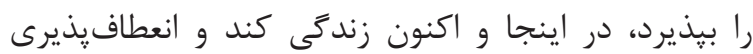

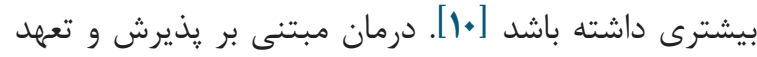

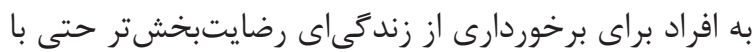

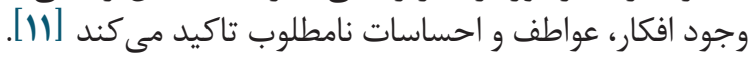

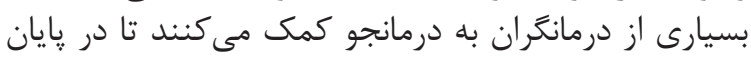

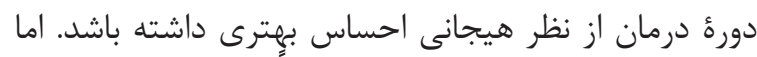

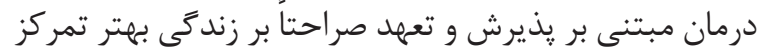

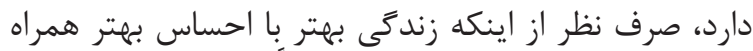

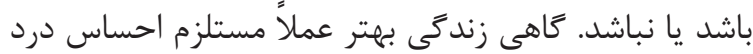

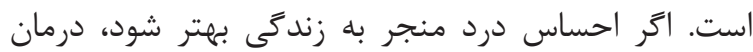

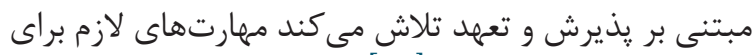

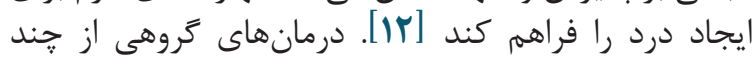

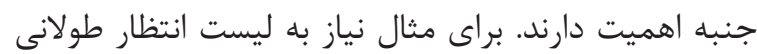

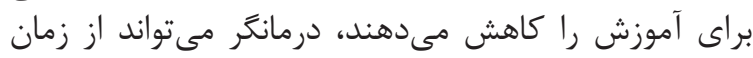

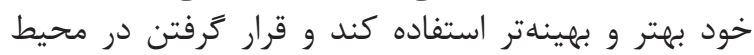

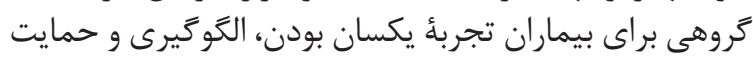

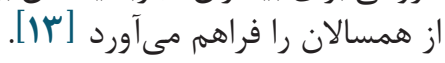

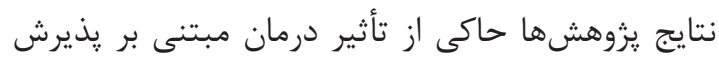

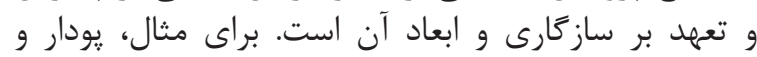

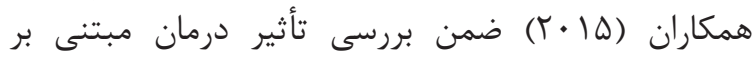

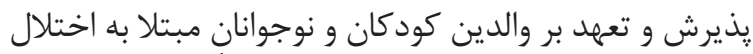

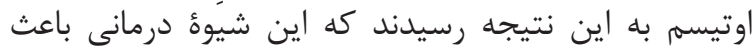

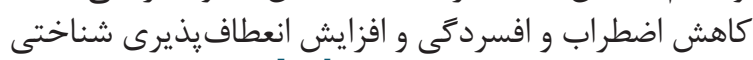

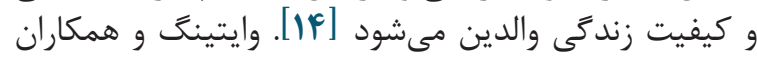

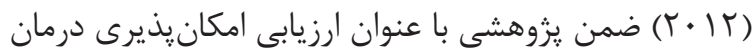

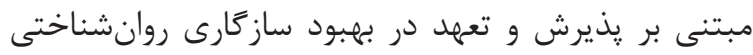

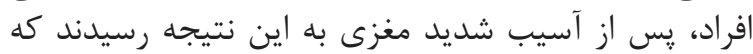

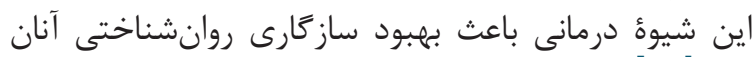

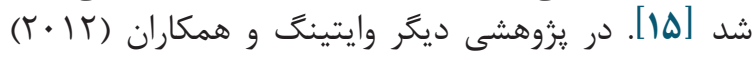

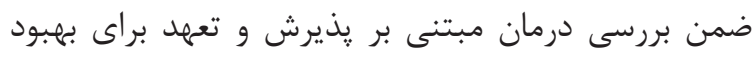

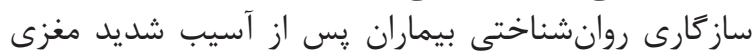

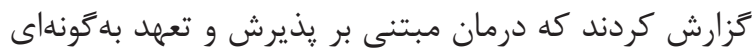

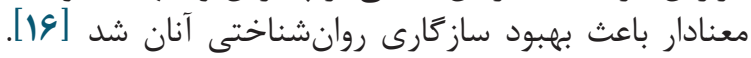

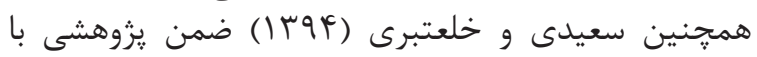




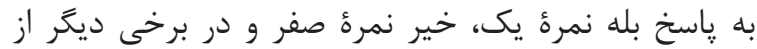

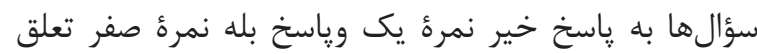

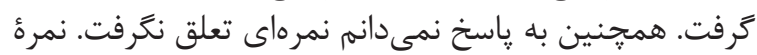

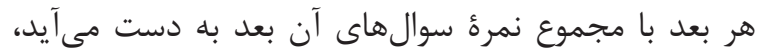

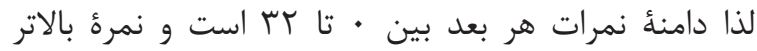

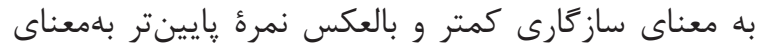

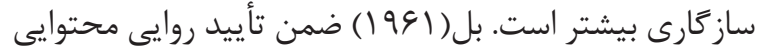

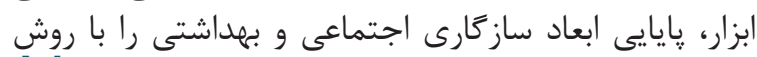

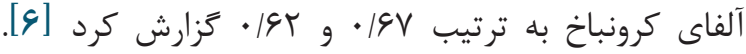

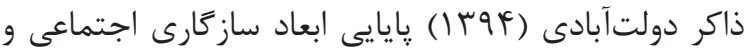

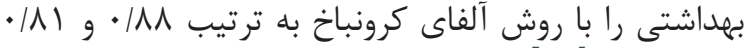

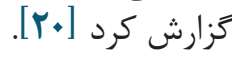

دادهها در سطح توصيفى با استفاده از شاخصهاى تر ترايش

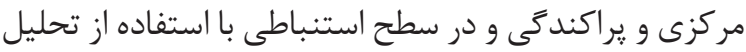

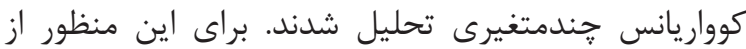

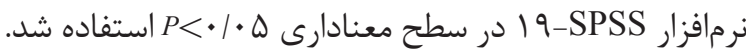

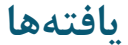

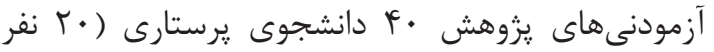

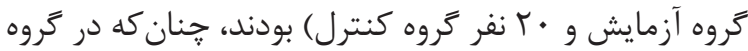

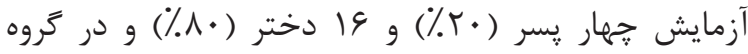

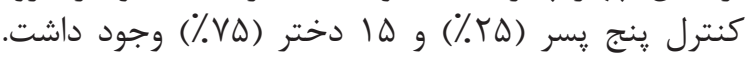

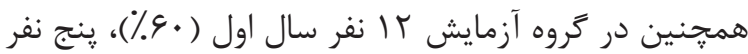

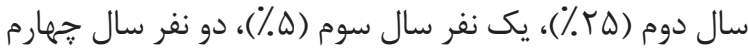

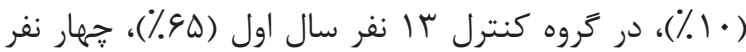

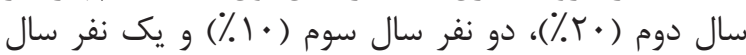

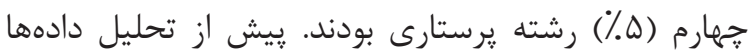

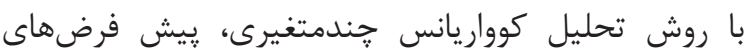

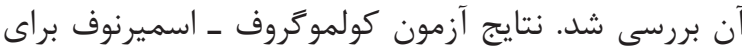

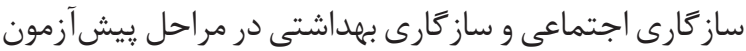

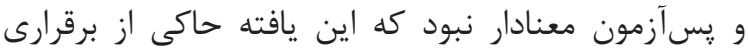

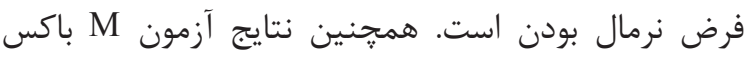

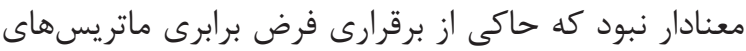

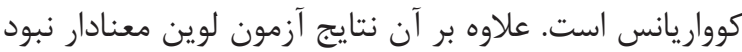

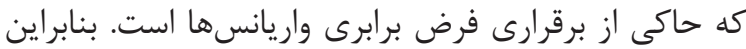

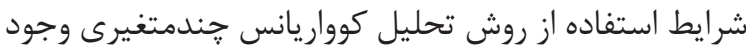

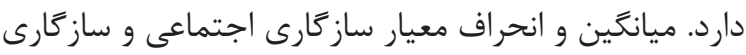

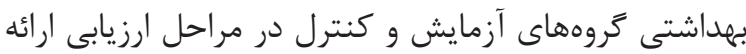

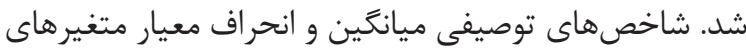

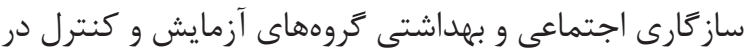

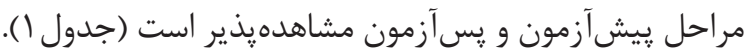

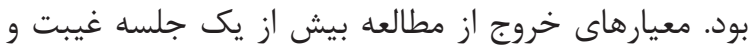

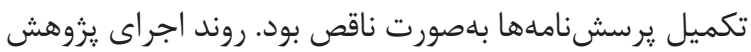

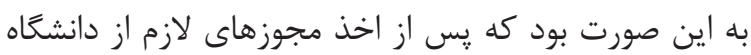

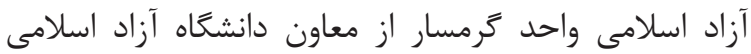

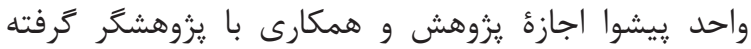

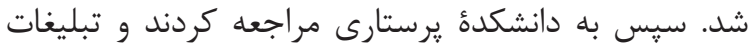

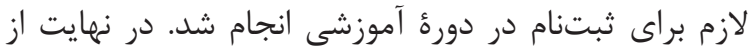

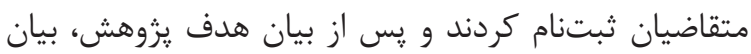

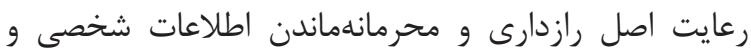

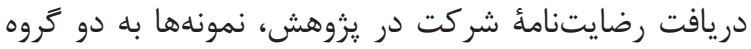

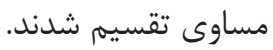

كروه آزمايش هشت جلسئ •9 دقيقهاى به شيوه كروهي

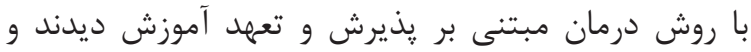

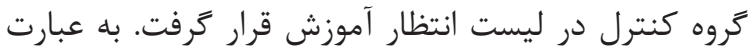

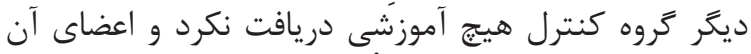

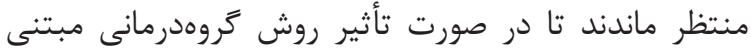

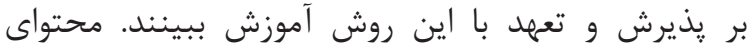

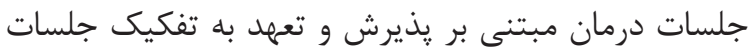

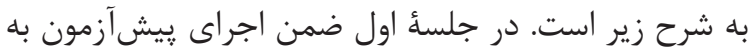

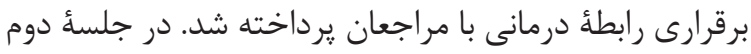

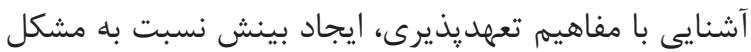

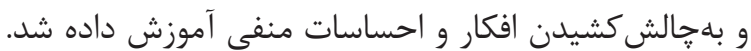

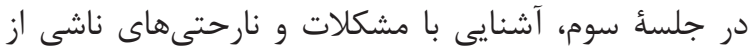

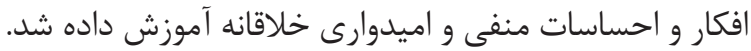

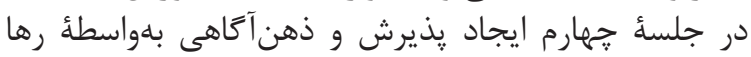

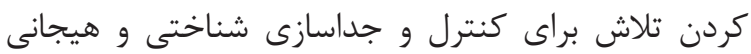

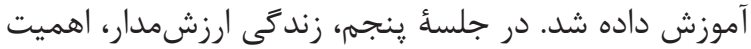

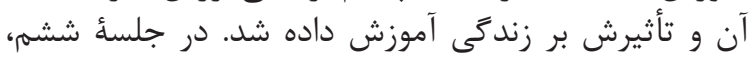

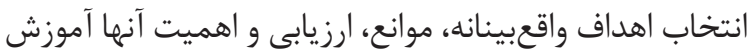

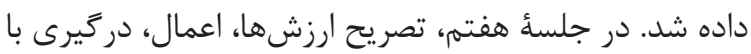

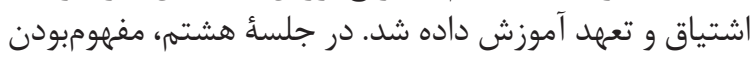

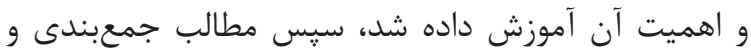

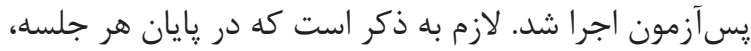

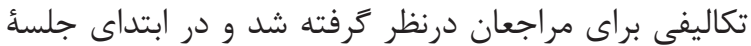

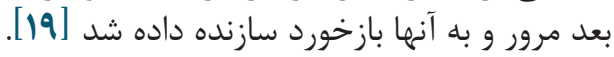

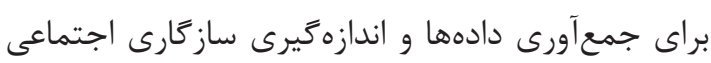

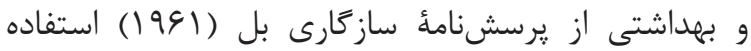

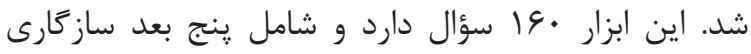

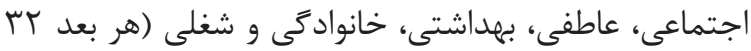

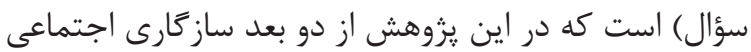

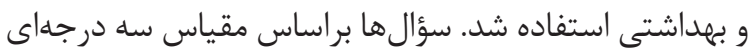

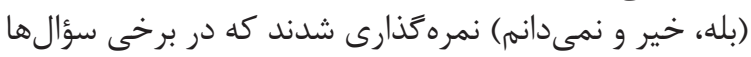

جدول شماره ا. ميانغين و انحراف معيار ساز كَارى اجتماعى و بهداشتى كروههاى آزمايش و كنترل در مراحل ارزيابى

\begin{tabular}{|c|c|c|c|c|c|c|c|c|}
\hline \multicolumn{4}{|c|}{ كروه كنترل } & \multicolumn{4}{|c|}{ كروه آزمايش } & \multirow{3}{*}{ كروهها } \\
\hline \multicolumn{2}{|c|}{ لـس بـ آزمون } & \multicolumn{2}{|c|}{ بيش آزمون } & \multicolumn{2}{|c|}{ لـ يس آزمون } & \multicolumn{2}{|c|}{ بيش آزمون } & \\
\hline SD & M & SD & $\mathbf{M}$ & SD & M & SD & $\mathbf{M}$ & \\
\hline$r / 9 \uparrow \wedge$ & $\left|\Delta / T^{\prime}\right|$ & $r / v \Delta \Delta$ & ID/DFT & r/NFT & $11 / \Delta / 1$ & $r / q \Delta f$ & $19 / 0 \cdot 1$ & سازَارى اجتماعى \\
\hline$r / / \Delta q$ & Ir/gr. & Tr/qT & $|r / A 9|$ & TIFT & 1./99. & $r / * \Lambda$ & IFIMGT & سازًَارى بهداشتى \\
\hline
\end{tabular}




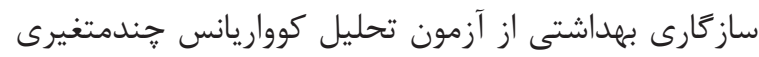

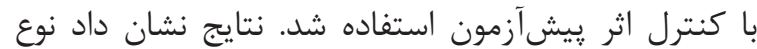

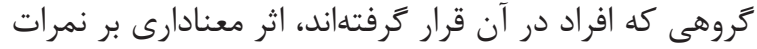

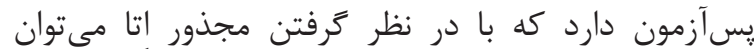

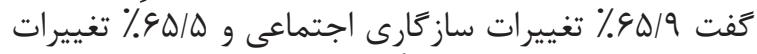

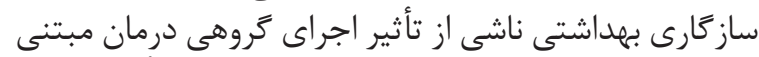

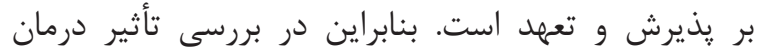

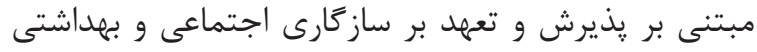

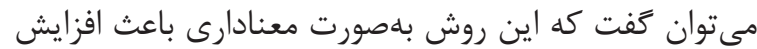

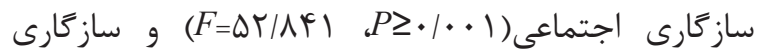

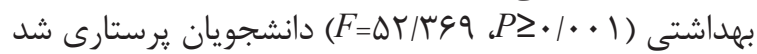

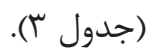

براى بررسى تأثير متغير مستقل (كروه درمانى مبتنى بر برائ

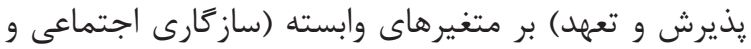

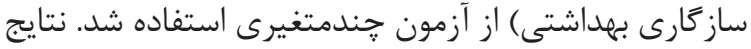

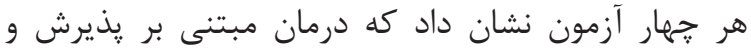

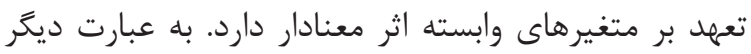

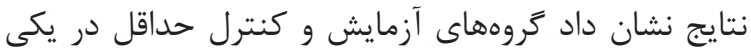

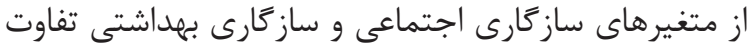

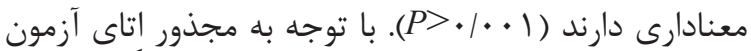

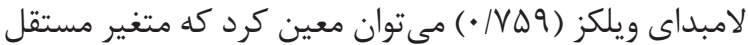

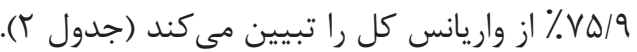

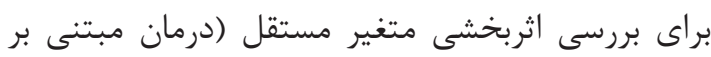

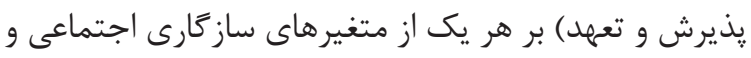

جدول شماره r. نتايج آزمون جندمتغيرى براى بررسى تأثير گروه درمانى مبتنى بر يذيرش و تعهد بر سازگارى اجتماعى و بهداشتى

\begin{tabular}{|c|c|c|c|c|c|}
\hline مجذور إتا & معنادارى & F آماره F & مقدار & آزمونها & اثر \\
\hline$\cdot / V \Delta 9$ & $\cdot 1 \cdot .1$ & $r \Delta / F \Delta F$ & .1919 & اثر پِيلايى & \multirow{4}{*}{ روش درمانى } \\
\hline - /VQQ & $\cdot 1 \cdot .1$ & $r \Delta / F \Delta F$ & $\cdot / r \mid \Lambda$ & لامبداى ويلكز & \\
\hline$\cdot / V \Delta q$ & $\cdot 1 \cdot \cdot 1$ & $r \Delta / F \Delta F$ & $r / l r v$ & اثر هاتلينغ & \\
\hline ./VDq & $.1 \cdot 1$ & $r \Delta / F \Delta F$ & $r / I r V$ & بزركترين ريشه روى & \\
\hline
\end{tabular}

جدول شماره ب. نتايج تحليل كوواريانس هندمتغيرى كروه درمانى مبتنى بر يذيرش و تعهد بر متغيرهاى سازكارى اجتماعى و سازكارى

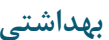

\begin{tabular}{|c|c|c|c|c|c|c|c|}
\hline مجذور إِا & معنادارى & F آماره F & مجذيانگين & لدرجه & مجذورات & منبع اثر & متغير هاى وابسته \\
\hline$\cdot|\Delta F|$ & $\cdot / \cdot 1$ & FN/TIF & IAV/rGS & 1 & INVITET & ي ريش آزمون & \multirow{5}{*}{ سازكارى اجتماعى } \\
\hline \multirow[t]{4}{*}{$\cdot 19 \Delta 9$} & $\cdot / . .1$ & $\Delta Y / A F \mid$ & $r \cdot \Delta / T Y F$ & 1 & $r \cdot \Delta / \mu F Y$ & تروه & \\
\hline & & & r/AMG & rv & IFr/var & واريانس خطا & \\
\hline & & & & r. & $\| \Lambda E / F \wedge F$ & واريانس كل & \\
\hline & & & & rq & $\Delta r g / 4 \wedge q$ & واريانس كل اصلاح شده & \\
\hline$\cdot / 499$ & $\cdot 1 \cdot .1$ & rN/FFt & $\mid r F / D \wedge q$ & 1 & $\mid r F / \Delta \wedge Q q$ & بيش آزمون & \multirow{5}{*}{ سازگارى بهداشتى } \\
\hline \multirow[t]{4}{*}{$\cdot \mid 9 \Delta \Delta$} & $\cdot 1 \cdot \cdot 1$ & $\Delta T / T \& Q$ & $I A r / T F V$ & 1 & $I A r / T F V$ & تروه & \\
\hline & & & $r / \Delta \cdot 1$ & rv & $1 \% q / \Delta F G$ & واريانس خطا & \\
\hline & & & & r. & $1 \cdot F N / T I F$ & واريانس كل & \\
\hline & & & & rq & FEV/FAT & واريانس كل اصلاح شده & \\
\hline
\end{tabular}

يوشش ادارة بهزيستى شد [IV]

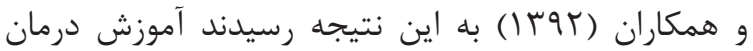

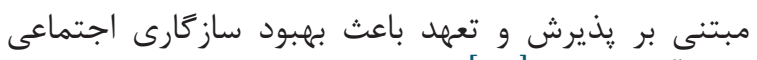

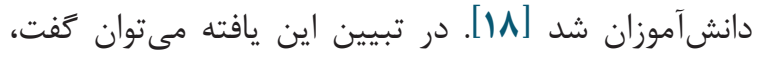

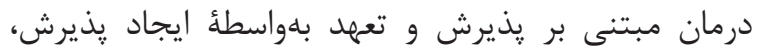

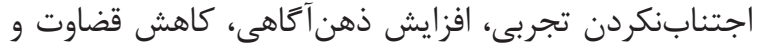

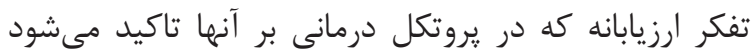

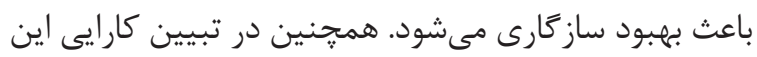

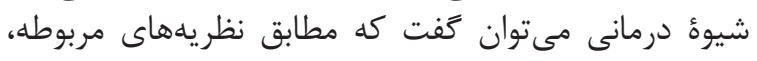

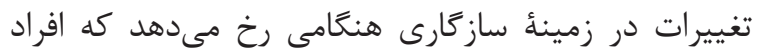

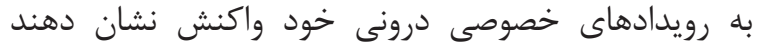

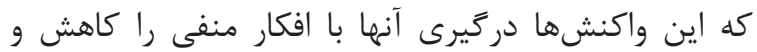

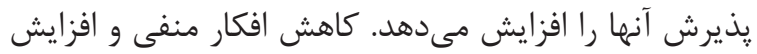

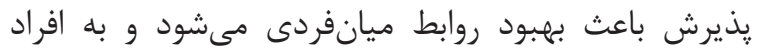
كمك مى كند تا مشاهده

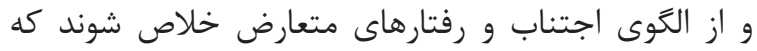

دوره ف شماره D - بهمن و اسفند وqها

\section{بحث}

مسئله سازكارى از مسائل مهمم دانشجويان است كه يكى داري

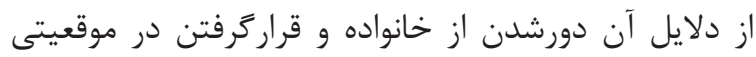

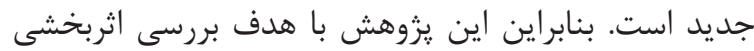

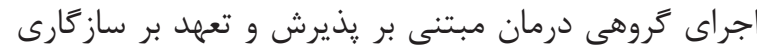
اجتماعى و بهداشتى دانشجويان يرستارى انجام شد.

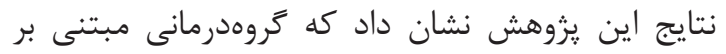

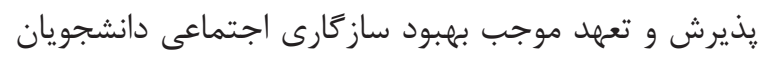

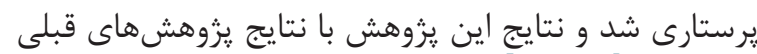

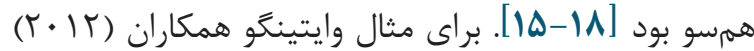

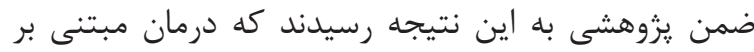

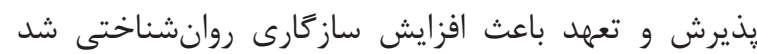

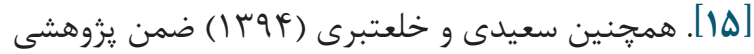

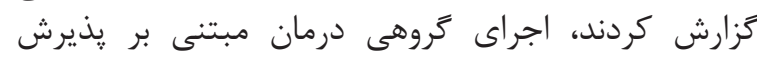

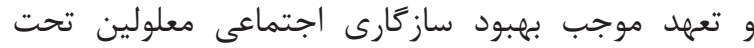




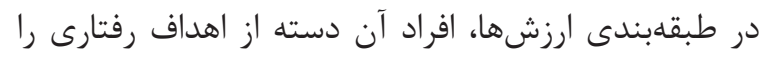

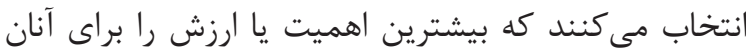

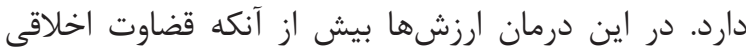

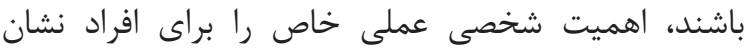

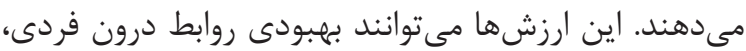

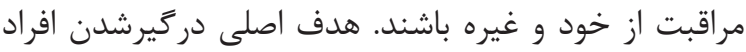

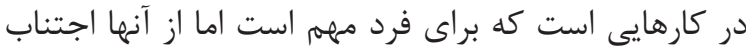

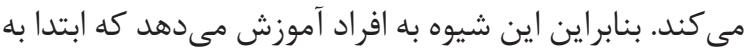

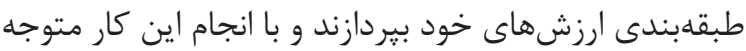

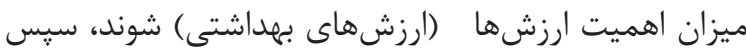

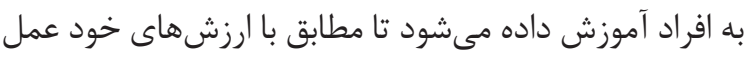

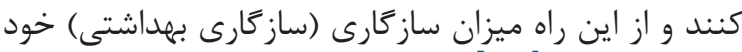

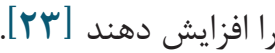

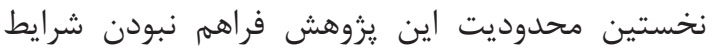

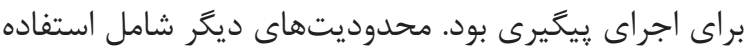

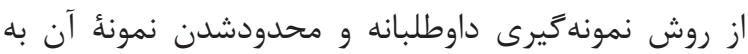

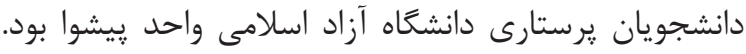

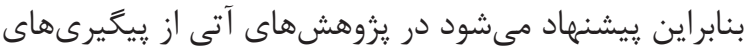

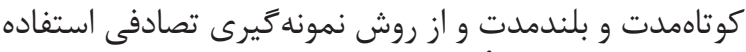

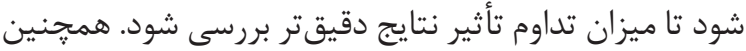

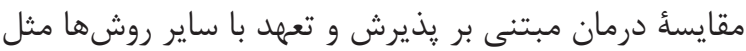

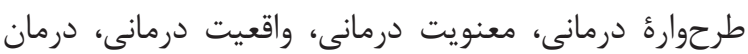

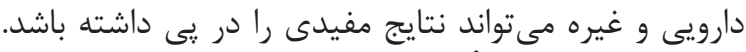

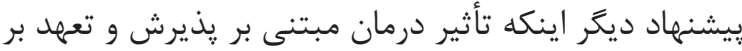

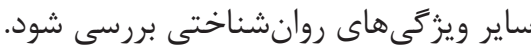

\section{نتيجهل}

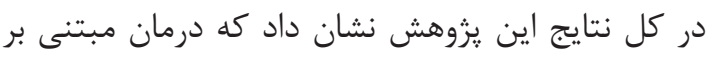

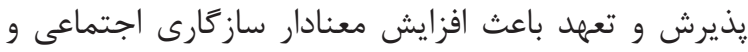

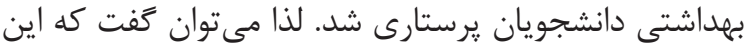

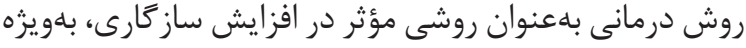

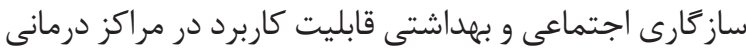

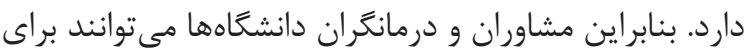

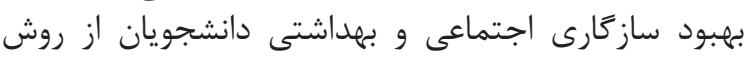

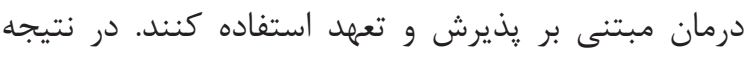

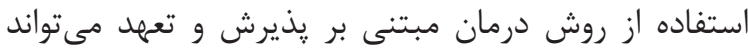

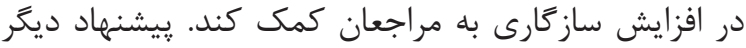

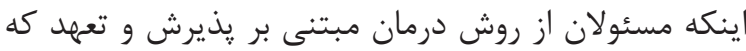

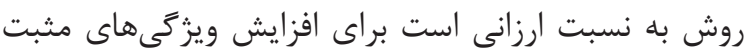

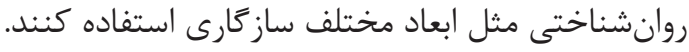

\section{سياسگزارى}

در يايان از كليئ مسئولين و دانشجويان برستارى دانشخاه

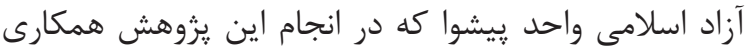

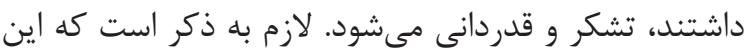

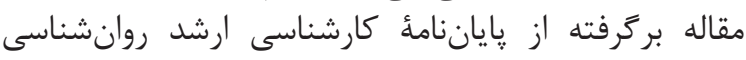

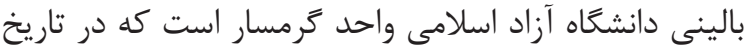
إس
اين عوامل مىتوانند در بهبود سازگًارى نقش مؤثرى داشته

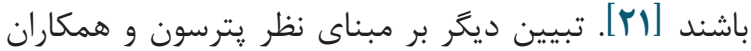

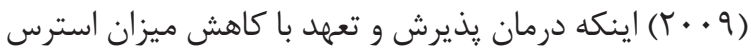

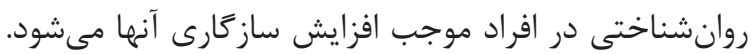

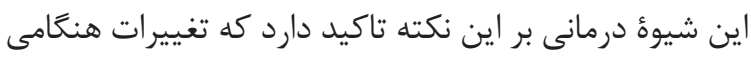

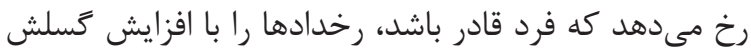

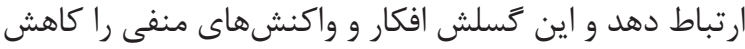

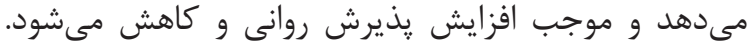

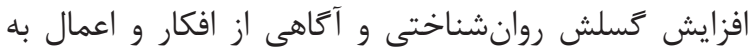

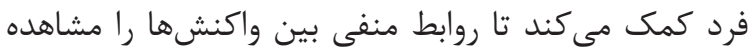

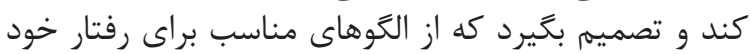

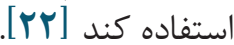

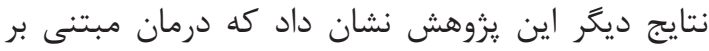

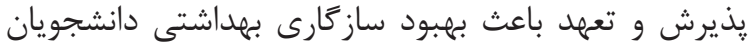

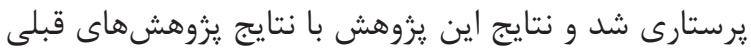

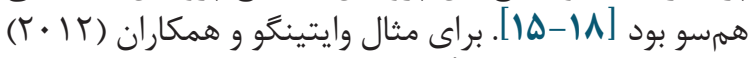

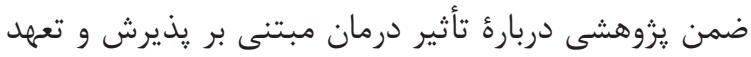

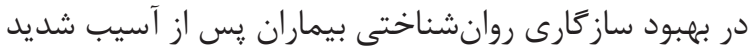

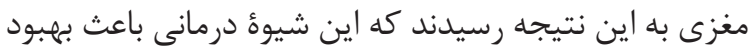

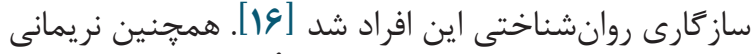

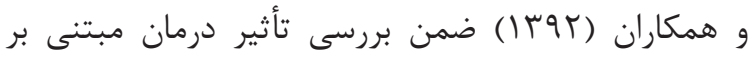

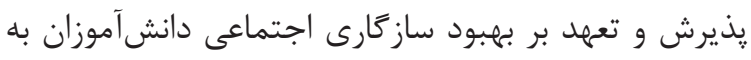

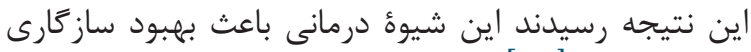

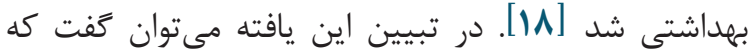

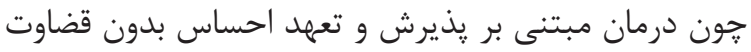

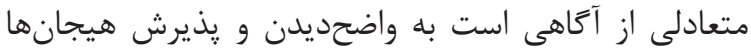

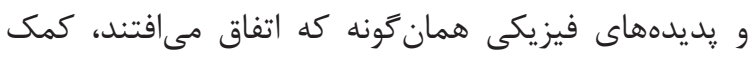

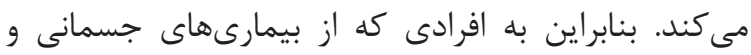

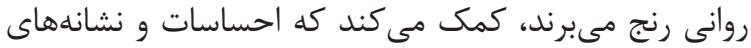

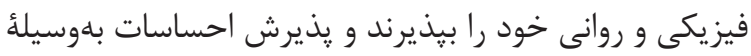

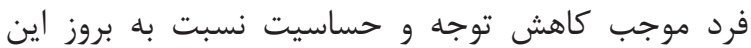

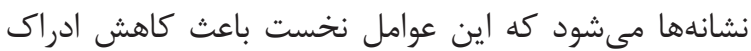

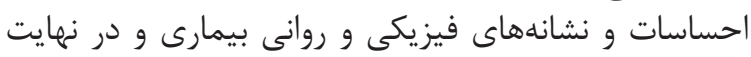

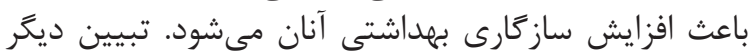

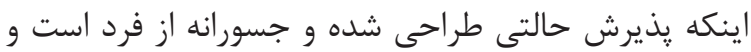

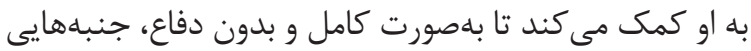

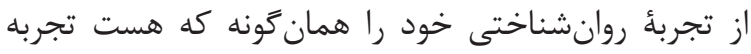

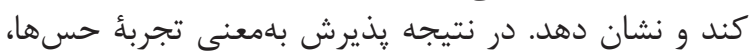

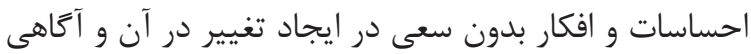

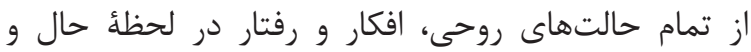

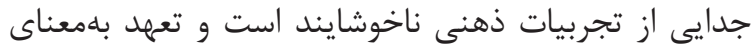

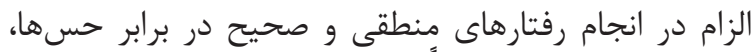

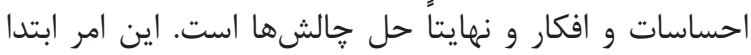

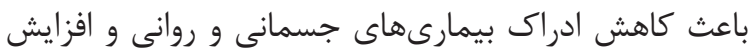

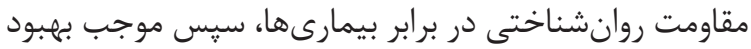

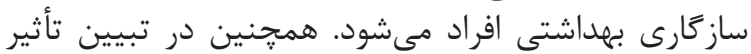

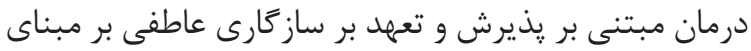

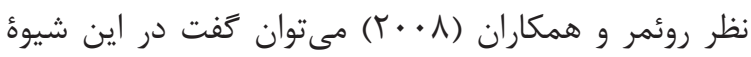

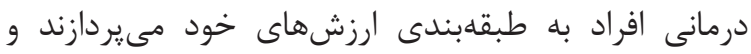




$$
\begin{aligned}
& \text { VA I I. اثربخشى كرومدرمانى مبتنى بر يذيرش و تعهد بر... } \\
& \text { تعارض در منافع } \\
& \text { بين نويسندكان در اين مطالعه تضاد منافعى وجود ندارد. }
\end{aligned}
$$

\section{References}

1. Ashoori J. Relationship between academic achievement and self-efficacy, critical thinking, thinking styles and emotional intelligence in nursing students. Sci J Hamadan Nurs Midwifery Fac. 2014;22(3):15-23.

2. Liu C, Zhao Y, Tian X, Zou G, Li P. Negative life events and school adjustment among Chinese nursing students: the mediating role of psychological capital. Nurs Edu Today. 2015;35(6):7549. https://doi.org/10.1016/j.nedt.2015.02.002 PMid:25702847

3. Wang C, Hatzigianni M, Shahaeian A, Murray E, Harrison LJ. The combined effects of teacher-child and peer relationships on children's social-emotional adjustment.J Sch Psyi chol. 2016;59:1-11. https://doi.org/10.1016/j. jsp.2016.09.003 PMid:27923438

4. Um M, Kim DH. The influence of adolese cent physical abuse on school adjustment in South Korea: The mediating effects of perceived parenting types. Children and Youth Serv Rev. 2015;59:89-96.https://doi.org/10.1016/j. childyouth.2015.10.016

5. Gois C, Akiskal H, Akiskal K, Figueira ML. Depressive temperament, distress, psychological adjustment and depressive symptoms in type 2 diabetes. J Affect Disord. 2012;143(13):1-4. https://doi.org/10.1016/j.jad.2012.05.028 PMid:22901331

6. Narimani M, YaghutiZargar H, Abolghasemi A, Nasrollahi A, Ahmadi V, et al. Study the effect of eye movement desensitization and reprocessing (EMDR) on the enhancement of social, affective and health adjustment of prisoners. J IlamUni Med Sci. 2014;22(1):52-8.

7. Zhao L, Xie Y, Wang J, Xu X. A performance asa sessment and adjustment program for air quality monitoring networks in Shanghai. Atmos Envie ron. 2015;122:382-92. https://doi.org/10.1016/j. atmosenv.2015.09.069

8. Khodayarifard M, Hejazi E, Hasaninezhad N. The effectiveness of Acceptance and commitment consultation (ACT) on self-efficacy and marital satisfaction substance using married women with children. J Appl Psychol Res. 2015;6(2):61-75.

9. Baruch B, Kanker J, Basch A. Acceptance and commitment therapy: enhancing the relationships. J Clin Case Study. 2019;8(3):241-57. https://doi.org/10.1177/1534650109334818

10. Ivanova E, Lindner P, Ly KH, Dahlin M, Vernmark K, et al.Guided and unguided acceptance and commitment therapy for social anxiety disorder and/or panic disorder provided via the internet and a smartphone application: a randomized controlled trial. J Anxiety Disord.2016;44:27-35. https://doi.org/10.1016/j.janxdis.2016.09.012 PMid:27721123

11. Eifert GH, Forsyth JP, Arch J, Espejo E, Keller $\mathrm{M}$, Langer D. Acceptance and commitment therapy for anxiety disorders: Three case studies exemplifying a unified treatment protocol. Cogn Behav Prac. 2009;16(4):368-85. https://doi. org/10.1016/j.cbpra.2009.06.001

12. Hayes SC, Levin ME, Plumb-Vilardaga J, Villatte JL, Pistorello J. Acceptance and commitment therapy and contextual behavioral science: examining the progress of a distinctive model of behavioral and cognitive therapy. Behav Ther. 2013;44(2):180-98. https://doi.org/10.1016/j. beth.2009.08.002 PMid:23611068 PMCid:PMC3635495

13. Skewes SA, Samson RA, Simpson SG, Van Vreeswijk M. Short-term group schema therapy for mixed personality disorders: a pilot study. Front Psychol. 2014;5:1592-8. PMid:25657631

14. Poddar S, Sinha VK, Urbi M. Acceptance and commitment therapy on parents of children and adolescents with autism spectrum disorders. Int $\mathrm{J}$ Educ Psychol Res. 2015;1(3):221-5. https://doi. org/10.4103/2395-2296.158331

15. Whiting D, Simpson G, Ciarrochi J, McLeod $\mathrm{H}$. Assessing the feasibility of Acceptance and Commitment Therapy in promoting psychological adjustment after severe traumatic brain injury. Brain Injury. 2012;26(4-5):588-9.

16. Whiting SL, Simpson GK, Mcleod HJ, Deane FP, Ciarrochi J. Acceptance and commitment therapy (ACT) for psychological adjustment after traumatic brain injury: reporting the protocol for a randomized controlled trial. Brain Impair. 2012;13(3):360-76. https://doi.org/10.1017/ BrImp.2012.28

17. Saeidi M, Khalatbari J. The effectiveness of acceptance/commitment therapy on adjustment and quality of life of disabled persons in welfare organization. J Educ Psychol. 2015;7(1):53-62.

18. Narimani M, Abbasi M, Abolghasemi A, Ahadi B. A study comparing the effectiveness of acceptance/ commitment by emotional regulation training on adjustment in students with dyscalculia. J Learn Disability. 2013;2(4):154-76.

19. Bach PA, Moran DJ. ACT in practice: case con- 
فاخته اديب و همكاران. IV9

ceptualization in acceptance and commitment therapy. Translate by Sara Kamali and NiloofarKianrad. Tehran: Arjmand Publisher; 2015.

20. Zaker SD. The relationship between body image with adjustment and loneliness among girl students of high school in Mashhad, [Dissertation]. Ghoochan Iran: Islamic Azad University of Ghochan Branch; 2015.

21. Rajabi Gh, Imani M, KhojasteMehr R, Beyrami $\mathrm{M}$, Beshlideh $\mathrm{K}$. The study of the efficacy of acceptance based behavior therapy and integrative behavioral couple therapy on women with distressed couples and general anxiety disorder. J
Res Behave Sci. 2014;11(6):600-19.

22. Peterson BD, Eifert GH, Feingold T,Davidson S. Using acceptance and commitment therapy to treat distressed couples: a case study with two couples. Cogn Behav Prac. 2009;16(4):430-42. https://doi.org/10.1016/j.cbpra.2008.12.009

23. Roemer L, Orsillo SM, Salters-Pedneault K. Efficacy of an acceptance-based behavior therapy for generalized anxiety disorder: Evaluation in a randomized controlled trial. J Consult Clin Psychol. 2008;76(6):1083-9. https://doi.org/10.1037/ a0012720 PMid:19045976 PMCid:PMC2596727 